\title{
Early traditional chinese medicine bundle therapy for the prevention of sepsis acute gastrointestinal injury in patients with severe sepsis
}

\begin{abstract}
To study the role of early traditional Chinese medicine bundle therapy on the prevention of sepsis-associated acute gastrointestinal injury (AGI). This was a multicenter, prospective cohort study of 296 consecutive patients with severe sepsis between 2013/3 and 2014/11; 150 patients received standard treatments (controls) and 146 received traditional Chinese medicine bundle therapy (intervention group) (herbal decoction gavage based on syndrome differentiation, acupuncture, application of mirabilite, and defecation mixture). D-lactic acid, diamine oxidase, endotoxin, gastrin, and intra-abdominal pressure were measured. AGI was classified into four levels. Compared with controls, D-lactic acid, diamine oxidase, endotoxin, gastrin, and intra-abdominal pressure in the intervention group were decreased and motilin was increased on day $7(\mathrm{P}<0.05)$. AGI incidence in the intervention group was lower than in controls $(\mathrm{P}<0.05)$. GIF scores of the intervention AGI II and III groups were lower than in controls $(\mathrm{P}<0.05)$. The APACHE II scores of intervention AGI II, III, and IV groups were lower than in controls $(\mathrm{P}<0.05)$. Compared with controls, mechanical ventilation time and ICU stay in the intervention group were shorter $(\mathrm{P}=0.011$ and $\mathrm{P}=0.035)$; and 28-day overall and AGI-attributed mortality were lower ( $\mathrm{P}=0.048$ and $\mathrm{P}=0.043$ ). For patients with severe sepsis, early traditional Chinese medicine bundle therapy could decrease AGI incidence and improve prognosis.
\end{abstract}

Volume 5 Issue 6 - 2017

\author{
Yifei Wang,' Yunhua Zhang,' Ronglin Jiang2 \\ 'ICU,Traditional Chinese Medical Hospital, China \\ ${ }^{2} \mathrm{CCU}$, Zhejiang Provincial Hospital of Traditional Chinese \\ Medicine, China
}

Correspondence: Ronglin Jiang, ICU, Zhejiang Provincial Hospital of Traditional Chinese Medicine Hangzhou 310000, Zhejiang Province, China, Tel 86-57I-13958095268, Email jiangronglin@।26.com

Received: January 0I, 1970 | Published: March 16, 2017

\section{Introduction}

Sepsis is a crucial clinical issue in critical care medicine. There are millions of new cases of sepsis every year globally and $>25 \%$ of these patients die. ${ }^{1}$ The immune function of patients with sepsis is depressed, which can easily lead to multiple organ dysfunction syndrome (MODS). ${ }^{2}$

The main manifestations of sepsis include gastrointestinal dysfunction or failure and gastrointestinal mucosa injury, which lead to the translocation of gastrointestinal bacteria and toxin, leading to septic shock 3,4. Furthermore, aggravation of the septic shock condition will lead to gastrointestinal mucosa function being further impaired, leading to a vicious circle. ${ }^{3}$

Studies have shown that early enteral nutrition (EN) support can improve the nutritional status and prognosis. ${ }^{5,6}$ In 2014, sepsis treatment guidelines suggested that patients with stable hemodynamics should receive EN support as soon as possible (within 48h). This early stage EN support could maintain the integrity of the intestinal mucosa, prevent bacterial translocation and organ dysfunction, and decrease the infection rate. ${ }^{7}$ However, in stressful situation, the gastrointestinal tract is depressed and its function is significantly weakened, resulting in difficult EN support. ${ }^{7-9}$ Impaired gastrointestinal motility will cause reflux and aspiration, increasing the risk of ventilator-associated pneumonia (VAP) and will lead to longer mechanical ventilation time, ICU stay, and total hospitalization. Some randomized controlled trials (RCT) found that 24-48h of EN treatment for ICU patients with trauma could significantly decrease mortality and hospitalization expenses. ${ }^{6,9}$

Treatments for sepsis include early resuscitation, antiinfection therapy, mechanical ventilation, nutritional support, immunoregulation, and symptomatic support. ${ }^{6}$ Prevention measures for gastrointestinal dysfunction include the use anti-acids to protect the gastrointestinal mucosal barrier, supplement glutamine, and microbial ecological agents. Based on the early goal-directed therapy $(\mathrm{EGDT})^{10}$ and bundle therapy theories proposed in recent years (herbal decoction gavage based on syndrome differentiation, acupuncture, application of mirabilite, and defecation mixture), ${ }^{11-13}$ the modern Chinese medicine differentiates syndrome, pays equal attention to strengthening body resistance and eliminating pathogenic factors, and proposes the concept of traditional Chinese medicine (TCM) bundle therapy from the concept of "wholism". According to the TCM theory, the six fu-organs function well when the gastrointestinal tract unobstructed. For patients with sepsis, TCM aims to maintain unobstructed daily stool elimination, good tolerance of EN, normal borborygmus, and normal intra-abdominal pressure. However, clinical data are still lacking about the efficiency of this approach in patients with sepsis.

Therefore, this study aimed to study the role of early traditional Chinese medicine bundle therapy on the prevention and treatment of sepsis after acute gastrointestinal injury (AGI). Patients received bundle intervention that included herbal decoction gavage according to syndrome differentiation, acupuncture at acupoints of Stomach Meridian of Foot-Yangming, application of mirabilite on the umbilicus, and enema with defecation mixture.

\section{Results}

\section{Characteristics of the patients}

From the 551 eligible patients, 296 patients were enrolled and included in the analysis (Figure 1). Table 1 presents the characteristics of these patients. There was no significant difference between the two groups for age, gender, APACHE II score, SOFA score, MODS score, source of infection, and reason of ICU admission (all $\mathrm{P}>0.05$ ). 


\section{Index of gastrointestinal function and intra-abdominal pressure}

Table 2 presents the laboratory indexes of gastrointestinal function of the patients. On day1, there was no significant difference between the two groups for D-lac, DAO, endotoxins, MTL, GAS, and intraabdominal pressure (all $\mathrm{P}>0.05$ ). On day3, compared with controls, the intra-abdominal pressure was lower in the intervention group $(\mathrm{P}<0.01)$ and MTL levels were higher $(\mathrm{P}<0.05)$, but there was no difference for D-lac, DAO, endotoxin, and GAS (all P $>0.05$ ). On day 7, D-lac, DAO, endotoxin, GAS, and intra-abdominal pressure were all lower in the intervention group than in controls (all $\mathrm{P}<0.05$ ), and MTL levels were higher $(\mathrm{P}<0.05)$.

\section{AGI incidence}

Within 7 days after admission, there were 88 patients with AGI in the control group and 69 patients in the intervention group. The incidence of AGI in the intervention group was lower than in the control group $(\mathrm{P}<0.05)$ (Table 3$)$.

\section{General condition of the patients}

There was no difference in the distribution of patients with AGI II, III, and IV between the intervention and control groups ( $\mathrm{P}>0.05)$. The GIF scores of the AGI II and III subgroups of the intervention group were significantly lower than the AGI II and III subgroups of the control group $(\mathrm{P}<0.05)$. APACHE II scores of the AGI II and III subgroups of the intervention group were lower than in the AGI II and III subgroups of the control group $(\mathrm{P}<0.05)$ (Table 4).

The patients were divided into three groups according to their APACHE II score at admission: $<10,10-20$, or $>20$. When comparing the AGI incidence, the AGI incidence of the $<10$ and 10-20 subgroups in the intervention group were lower than in the $<10$ and 10-20 subgroups of the control group $(\mathrm{P}<0.05)$ (Table 5).

The mechanical ventilation time and ICU stay in the intervention group were significantly shorter than in the control group (both $\mathrm{P}<0.05)$. The 28-day and AGI-related mortality in the intervention group were lower than in the control group $(\mathrm{P}<0.05)$ (Table 6) (Figure $1)$.

Table I Characteristics of the 296 patients with sepsis

\begin{tabular}{|c|c|c|c|}
\hline & Control group $(n=\mid 50)$ & Intervention group $(n=\mid 46)$ & $\mathbf{P}$ \\
\hline Age (years) & $76.4 \pm 13.9$ & $76.5 \pm 14.0$ & 0.927 \\
\hline Male, n (\%) & 109 (72.7\%) & $109(74.7 \%)$ & 0.792 \\
\hline SOFA score & $8.72 \pm|.5|$ & $9.02 \pm 1.40$ & 0.081 \\
\hline APACHE II score & $15.5 \pm 2.0$ & $15.9 \pm 2.0$ & 0.085 \\
\hline \multicolumn{4}{|l|}{ Original infection sites, n (\%) } \\
\hline Respiratory system & $80(53.3 \%)$ & $76(52.1 \%)$ & \multirow{6}{*}{0.787} \\
\hline Digestive System & $37(24.7 \%)$ & $29(19.9 \%)$ & \\
\hline Urinary system & 7 (4.7\%) & $10(6.9 \%)$ & \\
\hline Hematogenous infection & $9(6.0 \%)$ & $12(8.2 \%)$ & \\
\hline Intracranial infection & $5(3.3 \%)$ & $4(2.7 \%)$ & \\
\hline Other sites & $12(8.0 \%)$ & $15(10.3 \%)$ & \\
\hline \multicolumn{4}{|l|}{ Reason of ICU admission, $n$ (\%) } \\
\hline Respiratory failure & $69(46.0 \%)$ & 75 (5।.4\%) & \multirow{6}{*}{0.918} \\
\hline Circulatory failure & $20(13.3 \%)$ & $18(12.3 \%)$ & \\
\hline Nervous system (epilepsy, apoplexy) & $21(14.0 \%)$ & $16(11.0 \%)$ & \\
\hline Trauma & $17(11.3 \%)$ & $18(12.3 \%)$ & \\
\hline Others & $3(2.0 \%)$ & $2(1.4 \%)$ & \\
\hline Postoperative patient & $20(13.3 \%)$ & $17(11.6 \%)$ & \\
\hline \multicolumn{4}{|c|}{ Traditional chinese medicine syndrome } \\
\hline Qi activity stagnation & $52(34.7 \%)$ & 45 (30.8\%) & \multirow{4}{*}{0.773} \\
\hline Qi stagnation and blood stasis & $40(26.7 \%)$ & $36(24.7 \%)$ & \\
\hline Spleen-stomach weakness & $30(20.0 \%)$ & $35(24.0 \%)$ & \\
\hline Internal accumulation of damp-heat & $28(18.7 \%)$ & $30(20.6 \%)$ & \\
\hline
\end{tabular}

Table 2 Laboratory indexes of gastrointestinal function

\begin{tabular}{lllll}
\hline Indexes & Time point & $\begin{array}{l}\text { Control group } \\
(\mathbf{n}|=| \mathbf{5 0}) \\
(\mathbf{n} 3=\mid \mathbf{5 0}) \\
(\mathbf{n 7}=1 \mathbf{3 6})\end{array}$ & $\begin{array}{l}\text { Intervention group } \\
(\mathbf{n}|=| \mathbf{4 6}) \\
(\mathbf{n} 3=\mid \mathbf{4 6}) \\
(\mathbf{n 7} \mathbf{1 3 8})\end{array}$ & $\mathbf{P}$ \\
\hline \multirow{2}{*}{ D lactic acid $(\mathrm{mg} / \mathrm{L})$} & $508.25 \pm 285.03$ & $506.54 \pm 279.74$ & 0.960 \\
& Day I & $392.21 \pm 186.26$ & $373.01 \pm 163.78$ & 0.406 \\
& Day 3 & $377.79 \pm 204.93$ & $321.65 \pm 138.78$ & 0.044 \\
Diamine oxidase & Day 7 & $12.98 \pm 7.31$ & $12.97 \pm 7.31$ & 0.992 \\
$(\mathrm{U} / \mathrm{mL})$ & Day I & $10.65 \pm 5.32$ & $11.11 \pm 5.84$ & 0.530 \\
& Day 3 & $12.36 \pm 6.43$ & $10.09 \pm 4.55$ & 0.010 \\
Endotoxin $(\mathrm{eU} / \mathrm{ml})$ & Day 7 & $0.77 \pm 0.23$ & $0.78 \pm 0.21$ & 0.630 \\
& Day I & $0.68 \pm 0.18$ & $0.63 \pm 0.19$ & 0.097 \\
& Day 3 & $0.64 \pm 0.17$ & $0.59 \pm 0.17$ & 0.035 \\
\hline
\end{tabular}


Table Continued..

\begin{tabular}{|c|c|c|c|c|}
\hline Indexes & Time point & $\begin{array}{l}\text { Control group } \\
(n|=| 50) \\
(n 3=\mid 50) \\
(n 7=\mid 36)\end{array}$ & $\begin{array}{l}\text { Intervention group } \\
(n|=| 46) \\
(n 3=\mid 46) \\
(n 7=\mid 38)\end{array}$ & $\mathbf{P}$ \\
\hline \multirow{3}{*}{ Motilin (ng/L) } & Day I & $273.05 \pm 198.18$ & $268.55 \pm 198.18$ & 0.844 \\
\hline & Day 3 & $274.08 \pm 165.02$ & $324.12 \pm 201.30$ & 0.041 \\
\hline & Day 7 & $320.54 \pm 230.24$ & $423.02 \pm 318.69$ & 0.021 \\
\hline \multirow{3}{*}{ Gastrin (ng/L) } & Day I & $221.82 \pm 177.96$ & $216.42 \pm 177.63$ & 0.794 \\
\hline & Day 3 & $216.24 \pm 120.75$ & $183.79 \pm 1 \mid 3.08$ & $0.04 I$ \\
\hline & Day 7 & $214.32 \pm 121.37$ & $176.54 \pm 99.14$ & 0.037 \\
\hline \multirow{3}{*}{$\begin{array}{l}\text { Intra-abdominal pressure } \\
(\mathrm{mmHg})\end{array}$} & Day I & $9.77 \pm 4.02$ & $10.30 \pm 2.86$ & 0.193 \\
\hline & Day 3 & $11.23 \pm 4.11$ & $9.16 \pm 4.12$ & $<0.00$ I \\
\hline & Day 7 & $11.59 \pm 3.80$ & $10.28 \pm 2.61$ & 0.012 \\
\hline
\end{tabular}

Table 3 Comparison of AGI incidence

\begin{tabular}{llll}
\hline Groups & Control group $(\mathbf{n = 1 5 0 )}$ & Intervention group $(\mathbf{n}=\mid \mathbf{4 6})$ & $\mathbf{P}$ \\
\hline AGI (\%) & $88(58.67 \%)$ & $69(47.26 \%)$ & 0.04 I \\
No AGI (\%) & $62(41.33 \%)$ & $77(52.74 \%)$ & \\
\hline
\end{tabular}

Table 4 General condition of the patients

\begin{tabular}{|c|c|c|c|}
\hline & Control group $(\mathrm{n} 7=88)$ & Intervention group $(\mathrm{n} 7=69)$ & $\mathbf{P}$ \\
\hline \multicolumn{4}{|l|}{ AGI II } \\
\hline$n$ & $13(14.8 \%)$ & $18(26.1 \%)$ & 0.077 \\
\hline GIF score & $2.7 \pm 1.0$ & $2.2 \pm 0.9$ & 0.006 \\
\hline APACHE II score & $12.2 \pm 2.5$ & $11.3 \pm 2.0$ & 0.011 \\
\hline \multicolumn{4}{|l|}{ AGI III } \\
\hline $\mathrm{n}$ & $68(77.3 \%)$ & $48(69.6 \%)$ & 0.275 \\
\hline GIF score & $2.9 \pm 1.0$ & $2.4 \pm 1.0$ & 0.007 \\
\hline APACHE II score & $|I| \pm 2.0$. & $10.2 \pm 1.9$ & 0.013 \\
\hline \multicolumn{4}{|l|}{ AGI IV } \\
\hline$n$ & 7 (8.0\%) & $3(4.4 \%)$ & 0.358 \\
\hline GIF score & $4.7 \pm 0.7$ & $4.6 \pm 0.8$ & 0.552 \\
\hline APACHE II score & $13.7 \pm 1.9$ & $13.2 \pm 1.6$ & 0.023 \\
\hline
\end{tabular}

Table 5 Comparison of the AGI incidence according to APACHE II scores

\begin{tabular}{|c|c|c|c|}
\hline & APACHE II <I0, n (AGI incidence) & APACHE II I0-20, n (AGI incidence) & APACHE II >20, n (AGI incidence) \\
\hline Control group $(n=\mid 50)$ & $24(25.0 \%)$ & $70(5 \mathrm{I} .4 \%)$ & $56(82.1 \%)$ \\
\hline Intervention group $(n=\mid 46)$ & $21(0 \%)$ & $67(31.3 \%)$ & $58(82.8 \%)$ \\
\hline$P$ & 0.023 & 0.024 & 1.000 \\
\hline
\end{tabular}

Table 6 Comparison of prognosis between the two groups

\begin{tabular}{llll}
\hline Parameter & Control group $(\mathbf{n = I 5 0 )}$ & Intervention group $(\mathbf{n}=\mathbf{I 4 6})$ & $\mathbf{P}$ \\
\hline Mechanical ventilation time (days) & $10.4 \pm 3.7$ & $9.3 \pm 3.8$ & $0.01 \mathrm{I}$ \\
ICU stay (days) & $18.5 \pm 10.6$ & $14.2 \pm 7.9$ & 0.035 \\
28-day mortality & $43.3 \%$ & $31.5 \%$ & 0.048 \\
AGI-attributable mortality & $8.7 \%$ & $2.7 \%$ & 0.043 \\
\hline
\end{tabular}

\section{Discussion}

The aim of the present study was to study the role of early traditional Chinese medicine bundle therapy on the prevention and treatment of sepsis-associated AGI. Results showed that compared with controls, D-lactic acid, diamine oxidase, endotoxin, gastrin, and intraabdominal pressure in the intervention group was decreased on day 7; motilin was increased on day 7. AGI incidence in the intervention group was lower than in controls. GIF scores of the intervention AGI II and III groups were lower than in controls. The APACHE II scores of intervention AGI II, III, and IV groups were lower than in controls. Compared with controls, mechanical ventilation time in the intervention group was shorter; ICU stay was shorter; and 28-day overall and AGI-attributed mortality was lower.

Severe ischemia and anoxia observed in patients with sepsis often lead to gastrointestinal mucosal barrier dysfunction, allowing intestinal bacteria and endotoxin translocation. In the last decade, critical disease monitoring and organ function support technologies improved greatly, but mortality from sepsis is still as high as 20 $50 \%{ }^{1}$ The therapeutic approach of western medicine on sepsis gastrointestinal dysfunction is still limited to symptomatic treatment. On the other hand, the spleen-stomach theory of TCM pays attention to the spleen-stomach function, which is a crucial principle of syndrome differentiation. In the Treatise on Spleen and Stomach, Li indicated 
that internal impairment of the spleen and stomach will cause various diseases, suggesting the significant role of spleen and stomach on disease development. TCM believes that there cannot be any survival without stomach Qi. Some randomized clinical trials showed that in the first 24-48h, EN treatment for the patients with critical illness could significantly decrease mortality and hospitalization expenses of patients in the ICU. ${ }^{8,14}$ Reintam et al., ${ }^{15}$ suggested that because of the complex and various functions of the gastrointestinal tract, a single treatment would not improve the gastrointestinal tract in cases of severe illness.

\section{Survival proportions: Survival of Two groups}
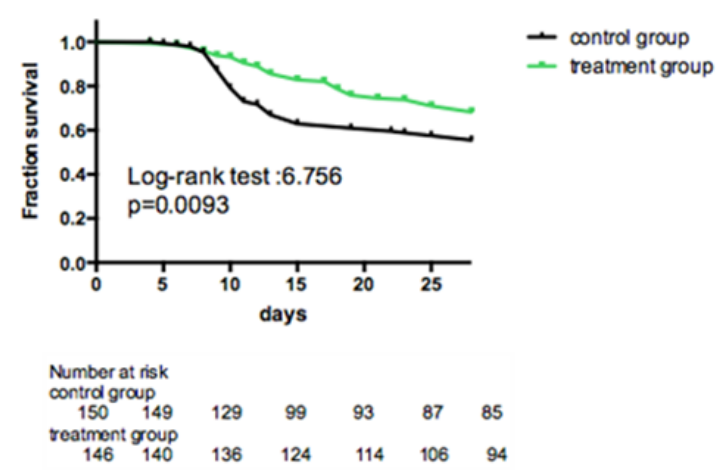

Figure I 28-day survival curve between the control group and intervention group.

TCM bundle therapy includes nasal feeding, tongbian mixture enema, umbilicus compress of mirabilite, acupuncture, and moxibustion. According to TCM, sepsis gastrointestinal dysfunction can be divided into four types: Qi activity stagnation, Qi stagnation and blood stasis, asthenia of spleen and stomach, and dampness and heat from interior. These syndromes are treated with Simo Yinzi, taohe chengqitang, six gentlemen decoction, and peptic powder, respectively. The main component of tongbian mixture enema is rheum officinale, which have various pharmacological actions and can protect gastrointestinal mucosal barrier, promote gastrointestinal motility, ${ }^{16}$ alleviate inflammatory reaction, decrease intestinal permeability, inhibit the absorption of endotoxin and growth of pathogenic bacterium, and improve cellular immunity and proliferation of lymphocyte to prevent GIF. ${ }^{17}$ The main components of mirabilite are sodium sulfate, calcium sulfate, and magnesium sulfate. Mirabilite has strong water absorbing power and some effects including against diarrhea and detumescence, as well as diuretic effects. External application of mirabilite on the abdomen can withdraw the ascites, ease the burden of abdominal distension and intestinal tract, promote the detumescence of enterocoelia swollen organs, and is beneficial to the control of inflammation and recovery of intestinal functions. ${ }^{18}$ Acupuncture at acupoints of Stomach Meridian of Foot-Yangming has some effects including regulating spleen and stomach, invigorating spleen-reinforcing Qi, clearing and activating the channels and collaterals, strengthening body resistance and eliminating evil, dual-direction regulation of nerve-internal secretionimmune systems, inhibition of systemic inflammatory response, and few adverse reactions. ${ }^{19}$ A previous study showed that acupuncture at ST36 and EA could improve the intestinal mucosal barrier in sepsis. ${ }^{13}$

DAO is an enzyme with high activity that exists in the intestinal mucosal epithelium. Under pathological states, intestinal mucosal cells suffer from an ischemic injury, and DAO is released into circulation; it is therefore a marker of intestinal damage. D-lac is a metabolite of intestinal bacteria fermentation and endotoxin is a direct marker of the presence of Gram-negative intestinal bacteria. Normally, D-lac and endotoxins cannot get through the intestinal mucosal barrier; therefore, any increase of their blood levels indicates changes of intestinal permeability or barrier function, and D-lac and DAO are early diagnosis indicators of gastrointestinal mucosal barrier function injury. ${ }^{20}$ The present study showed that on the 3rd day after admission, the levels of D-lac, DAO, and endotoxin in the intervention group were lower than in the control group, but without reaching statistical significance $(\mathrm{P}>0.05)$, but on the 7th day, levels of D-lac, DAO, and endotoxin in the intervention group were significantly lower than in the control group, indicating that gastrointestinal mucosal barrier function injury was alleviated.

MTL and GAS are key gastrointestinal hormones involved in gastrointestinal motility. MTL is an excitant gastrointestinal hormone that can regulate the cyclic activity of interdigestive myoelectric complexes, maintain normal peristalsis of stomach and small intestine, and prevent the over-growth of gastrointestinal bacteria. ${ }^{21}$ GAS is secreted by G-cells in the antrum and duodenum, and can promote gastric acid secretion. In patients with severe sepsis who are in a stress state, the gastrointestinal blood supply is reduced suddenly, acid-base balance is impaired, GAS secretion increases constantly and stress ulcer hemorrhage is easily induced. The present study showed that on the 3rd and 7th day after admission, MTL in the intervention group was higher than in the control group, and GAS in the intervention group was lower than in the control group, strongly suggesting that TCM bundle therapy can significantly improve the secretion of gastrointestinal hormones in patient with severe sepsis and should help maintaining the gastrointestinal functions.

In recent years, some studies demonstrated that acupuncture could stimulate the release of peptidergic neurotransmitters including MTL, and activate the peptidergic neural pathway to play a role and promote the recovery of inhibited gastric myoelectric activity. ${ }^{22}$ The present study also found that at on the 3rd and 7th days, the intra-abdominal pressure in the intervention group was obviously lower than in the control group and that the incidence of abdominal compartment syndrome was decreased, leading to improved gastrointestinal function of patient with severe sepsis.

The present study showed that after TCM bundle intervention, the AGI incidence over 7days was significantly decreased. GIF and APACHE II scores of patients with AGI II and III in the intervention group were lower than in the control group, but without difference in AGI IV. Therefore, TCM bundle therapy might effectively improve light and moderate gastrointestinal dysfunction. However, with aggravating disease, the factors influencing gastrointestinal function are increased and probably overcome the effects of TCM. Thus, the present study indicated that there was no difference of GIF and APACHE II scores for patients with AGI IV. TCM treatments should be studied further to determine more effective approaches.

Fang et al., ${ }^{23}$ found that the APACHE II, SOFA, and GIF scores of patients with MODS in the rheum officinale intervention group were obviously lower than in the standard group, and that the prognosis was better. In the present study, TCM bundle therapy was used to prevent AGI and good effects were achieved, as reflected by the lower GIF and APACHE II scores. After analysis of patients with various APACHE II scores, the study showed that TCM was more effective for patients with low APACHE II scores at admission. Zhang et al., ${ }^{24}$ considered that AGI levels were significantly associated with 28-day mortality and APACHE II score. In the present study, compared with controls, APACHE II scores in the intervention group were lower, mechanical ventilation time and stay in ICU was shorter, and 28-day mortality 
and gastrointestinal-related mortality were lower. These data strongly suggest that TCM bundle intervention could reduce disease severity of patients with severe sepsis. A recent meta-analysis revealed that addition of TCM added benefits in patients with sepsis. ${ }^{12}$

The present study is not without limitations. Indeed, the sample size was small. In addition, the patients were highly selected. Furthermore, even if a number of studies have shown the beneficial effects of TCM, the exact mechanisms of action are still mostly unknown. Additional studies are necessary to characterize TCM and improve its efficacy.

In conclusion, for patients with severe sepsis, early TCM bundle therapy might help avoiding AGI and improve prognosis.

\section{Methods}

\section{Study design}

This was a prospective multicenter study of 551 consecutive patients with severe sepsis treated at the ICUs of the Zhejiang Provincial Hospital of Traditional Chinese Medical, Tongde Hospital of Zhejiang Province, Xinhua Hospital of Zhejiang Province, and Hangzhou Traditional Chinese Medical Hospital between March 2013 and November 2014. The study protocol was approved by the Ethics Committee of each participating hospital. Informed consent forms were signed by the legal representatives of the patients.

\section{Patients}

All patients have been diagnosed with severe sepsis according to the diagnostic criteria of severe sepsis of the International Guidelines for Management of Severe sepsis and Septic Shock 2012. ${ }^{1}$ The patients had to meet any of the following criteria ${ }^{25}$ and be accompanied with organ dysfunction and/or tissue hypoperfusion caused by sepsis: 1) hypotension caused by sepsis; 2) lactic acid levels are higher than the normal value; 3 ) although given enough fluid resuscitation, urine volume is $<0.5 \mathrm{ml} / \mathrm{kg} / \mathrm{h}$ for at least 2 hours; 4) non-pneumonic acute lung injury and $\mathrm{PaO} 2 / \mathrm{FiO} 2<200 \mathrm{mmHg}$; 5) pneumonic acute lung injury and $\mathrm{PaO} 2 / \mathrm{FiO} 2<200 \mathrm{mmHg}$; 6) serum creatinine $>176.8 \mu \mathrm{mol} / \mathrm{L}(2.0 \mathrm{mg} / \mathrm{dl}) ; 7)$ bilirubin $>34.2 \mu \mathrm{mol} / \mathrm{L}(2 \mathrm{mg} /$ dl); (8) platelets $<100,000 / \mu \mathrm{L}$; and/or 9) blood coagulation disorders (international normalized ratio (INR) $>1.5$ ).

Exclusion criteria were: 1) ICU stay $<3$ days; 2) aged $\leq 18$ years; 3 ) pregnant or lactating women; 4) terminal malignant tumor; 5) Marshall score $\geq 20 ; 6$ ) esophagus, stomach, or intestinal medical and surgical histories or primary injury to the gastrointestinal tract when admitted at the ICU; 7) with AGI at admission; or 8) unstable hemodynamics or significantly abnormal coagulation function after 2 days at the ICU that cannot be treated with TCM nasal feeding (APTT $>2$ times the upper limit of normal).

\section{Allocation}

Patients with stable hemodynamics (mean arterial pressure (MAP) $\geq 65 \mathrm{mmHg}$ ) were immediately assigned to one of two groups, based on the willingness of the patients to receive TCM: 156 patients refused TCM (controls) and 175 accepted TCM (intervention group). The patients in the intervention group received TCM bundle therapy.

\section{Treatments}

In the control group, according to the International Sepsis Treatment Guide 2012, all patients received early EGDT for shock patients, respiratory and circulation support, active anti-infection therapy, and maintenance of electrolyte and acid base balance. Cases with stable hemodynamic received early EN support ${ }^{26,27}$ as soon as possible. A naso-intestinal tube was indwelled in each patient. EN was performed with continuous infusion by nutrition pump. Parenteral nutrition (PN) support within a week was avoided as much as possible.

In the intervention group, patients received TCM bundle therapy in addition to the treatments of the control group. According to the Diagnostics of Traditional Chinese Medicine ,28 the patients were divided into four types according to syndrome differentiation of TCM: I) Qi activity stagnation ( $\mathrm{n}=45)$, which was treated with Simo Yinzi; II) Qi stagnation and blood stasis $(\mathrm{n}=36)$, which was treated with taohe chengqitang; III) asthenia of spleen and stomach $(\mathrm{n}=35)$, which was mainly treated with the six gentlemen decoction; and VI) dampness and heat from interior $(\mathrm{n}=30)$, which was mainly treated with peptic powder. All decoctions were made to $80 \mathrm{~mL}$ decoction in decoction room. The patients received one dose $(80 \mathrm{~mL})$ each day, by nasal feeding, in two doses of $40 \mathrm{~mL}$. If syndrome type changed during treatment, the treatment was adjusted.

The patients in the intervention group were treated with acupuncture at the acupoints of Stomach Meridian of Foot-Yangming: st 36, Tien chu, Shangjuxu, and Xiajuxu. The location standard of acupoint was made referring to the National Standard Nomenclature and Location of Acupuncture Points 2006 (GB/T12346- 2006). The operating doctors had to be certified in acupuncture specialty. The needle was retained for 30 minutes after developing needle sensation, one time a day. Acupuncture was not performed for patients with local skin infection and anabrosis around the acupoints.

For external application of mirabilite to the abdomen, 500g of mirabilite were mashed into granules and put in a sand bag (about $25 \times 20 \mathrm{~cm}$ ). The sand bag was spread to middle and upper abdomen. The bag was replaced when it became hard or crystallized. The bag was applied 3times per day, 2hours each time.

The defecation drug mixture was made by the Zhejiang Provincial Hospital of Traditional Chinese Medical. Each liter of mixture contained $8.2 \mathrm{~g}$ of Chinese honey locust and $160 \mathrm{~g}$ of honey. Fifty $\mathrm{mL}$ of the mixture was slowly infused in the rectum and retained for $20 \mathrm{~min}$, once a day. This was not performed for patients with severe diarrhea.

The details of drug formulations are as follow. Simo Yinzi: $6 \mathrm{~g}$ of lignum aquilariae sinensis, $6 \mathrm{~g}$ of root of combined spicebush root, $6 \mathrm{~g}$ of south common vladimiria root, and $6 \mathrm{~g}$ of fructus aurantii. Taohe chengqitang: $12 \mathrm{~g}$ of semem persicae, $12 \mathrm{~g}$ of rheum officinale, $12 \mathrm{~g}$ of prepared Licorice root, $6 \mathrm{~g}$ of cassia twig, and $6 \mathrm{~g}$ of mirabilite. Six gentlemen decoction: $6 \mathrm{~g}$ of ginseng, $12 \mathrm{~g}$ of bighead atractylodes rhizome, $6 \mathrm{~g}$ of poria cocos, $4 \mathrm{~g}$ of liquorice, $5 \mathrm{~g}$ of orange peel, $6 \mathrm{~g}$ of roast pinellia ternata, $5 \mathrm{~g}$ of fructus amomi, and $4 \mathrm{~g}$ of radix aucklandiae. Peptic powder: $12 \mathrm{~g}$ of rhizoma atractylodis, $9 \mathrm{~g}$ of mangnolia officinalis, $6 \mathrm{~g}$ of orange peel, and $3 \mathrm{~g}$ of liquorice.

\section{Data collection and laboratory examinations}

Age, gender, source of infection, and reason of ICU admission were collected at admission. Sequential organ failure assessment (SOFA), APACHE II, and MODS occurring within 24hours were recorded. Blood was sampled on the $1 \mathrm{st}$, 3rd, and 7th days after admission to determine the levels of D-lactic acid (D-lac), diamine oxidase (DAO), endotoxin, motilin (MTL), gastrin (GAS), and intraabdominal pressure. The 28-day survival was collected.

D-lac, DAO, endotoxin, MTL, GAS, prealbumin, and albumin levels were determined at a third class A hospital. DAO was determined 
by spectrophotometry, while D-lac was determined with modified enzymatic spectrophotometry (Nanjing Jiancheng Bioengineering Institute, Nanjing, China). The quantitative determination of endotoxin was performed with a limulus kit (Sigma, St Louis, MO, USA). GAS was determined with the GAS radioimmunoassay kit (China Institute of Atomic Energy, Beijing, China). MTL was determined with the motilin radioimmunoassay kit (Shanghai Kerui Biotechnology Center, Shanghai, China). GAS and MTL were determined with ELISA. The amount of EN amount was determined as the fed EN solution volume through minus the gastric residual volume, which was determined by extracting per 6hours.

Foley catheters were connected with urine storage bag and piezometer tube for the patients in supine position. Then, the bladder was emptied, the urine storage bag was closed and filled with $20 \mathrm{~mL}$ of sodium chloride $(0.9 \%)$ through the piezometer tube. The piezometer tube and transducer were connected, the distal end was open, and after zero adjustment with the pubic symphysis taken as the zero, the intravesical pressure was determined as the reference of intraabdominal pressure.

AGI, as proposed by the European Society Intensive Care Medicine (ESICM) in 2012, ${ }^{29}$ indicates gastrointestinal dysfunction caused by acute illness of patients with critical illness. Depending on the severity, AGI could be divided into four levels: Level I indicates that there are risks of gastrointestinal dysfunction and temporary gastrointestinal symptoms with definite reasons; Level II indicates gastrointestinal dysfunction (GID) ${ }^{30}$ in which gastrointestinal digestion-absorption function cannot satisfy the need for nutrient substance and water, without the influence on the general physical condition of patient. Level III indicates gastrointestinal failure (GIF), in which the gastrointestinal function fails to recover and general physical condition is not improved although some intervention treatments are taken. Level IV indicates that GIF and other organs are severely impacted with MODS and shock. Due to the reversibility and spontaneous recovery of with AGI I, the cases with AGI II, III and IV were grouped as being diseased. Reintam et al., ${ }^{31}$ proposed three typical gastrointestinal syndromes of GIF: feeding intolerance syndrome (FI), gastrointestinal hemorrhage $(\mathrm{GIH})$, and paralytic ileus (PI).

According to the standard for GIF evaluation by Reintam et al., ${ }^{32} \&$ Sun et al. ${ }^{33} 0$ indicates that the gastrointestinal function is normal; 1 indicates that the EN amount is less than $50 \%$ of necessary amount or the patients that cannot take food 3days after abdominal operation; 2 indicates gastrointestinal nutrition intolerance or intraabdominal hypertension; 3 indicates gastrointestinal nutrition intolerance with intra-abdominal hypertension; and 4 indicates abdominal compartment syndrome.

\section{Adverse events}

If an adverse event was suspected of resulting from the Chinese medicine intervention, the investigator had to fill the form of adverse event, and drop the patient out of the study.

\section{Statistical analysis}

Continuous data ware presented as mean \pm standard deviation and was analyzed with the independent-samples $t$ test or ANOVA with the Tukey's post hoc test, as appropriate. Categorical data are presented as frequencies and were analyzed with the Fisher Exact test. Survival was analyzed according to the Kaplan-Meier method and was analyzed with the Log rank test. Statistical analysis was performed with SPSS 21.0 (IBM, Armonk, NY, USA). Two-sided P-values $<0.05$ were considered statistically significant.

\section{Acknowledgements}

Major research plan of disease prevention and treatment of traditional Chinese Medicine in Zhejiang Province(No.2012ZGG001).

\section{Author contributions}

YF.W and YH.Z significantly contributed to the study design and RL.J contributed to concept. YF.W and YH.Z drafted the manuscript. All authors reviewed and approved the manuscript.

\section{Conflicts of interest}

Author declares there are no conflicts of interest.

\section{Funding}

None.

\section{References}

1. Dellinger RP, Levy MM, Rhodes A, et al. Surviving Sepsis Campaign: international guidelines for management of severe sepsis and septic shock, 2012. Crit Care Med. 2013;41(2):580-637.

2. Griffiths B, Anderson I D. Sepsis, SIRS and MODS. Surgery. 2009;27(10):446-449.

3. Fink MP. Gastrointestinal mucosal injury in experimental models of shock, trauma, and sepsis. Crit Care Med. 1991;19(5):627-641.

4. Schmidt C, Lautenschläger C, Petzold B, et al. Confocal laser endomicroscopy reliably detects sepsis-related and treatmentassociated changes in intestinal mucosal microcirculation. Br J Anaesth. 2013;111(6):996-1003.

5. Puleo F, Arvanitakis M, Van Gossum A,et al. Gut failure in the ICU. Seminars in respiratory and Crit Care Med. 2011;32(5):626-638.

6. Gerlach H, Toussaint S. Managing septic shock. F1000 medicine reports. 2010 .

7. Doig GS, Heighes PT, Simpson F, et al. Early enteral nutrition, provided within $24 \mathrm{~h}$ of injury or intensive care unit admission, significantly reduces mortality in critically ill patients: a meta-analysis of randomised controlled trials. Intensive Care Med. 2009;35(12):2018-2027.

8. Doig GS, Heighes PT, Simpson F, et al. Early enteral nutrition reduces mortality in trauma patients requiring intensive care: a meta-analysis of randomised controlled trials. Injury. 2011;42(1):50-56.

9. Manba N, Koyama Y, Kosugi S, et al. Is early enteral nutrition initiated within 24 hours better for the postoperative course in esophageal cancer surgery? J Clin Med Res. 2014;6(1):53-58.

10. Rivers, E, Nguye B, Havstad S, et al. Early goal-directed therapy in the treatment of severe sepsis and septic shock. $N$ Engl J Med. 2001;345:1368-1377.

11. Shiramizo SC, Marra AR, Durão MS, et al. Decreasing mortality in severe sepsis and septic shock patients by implementing a sepsis bundle in a hospital setting. PloS one. 2011;6(11):e26790.

12. Liang X, Zhou M, Ge XY, et al. Efficacy of traditional Chinese medicine on sepsis: a systematic review and Meta-Analysis. Int J Clin Exp Med. 2015;8(11):20024-20034.

13. Zhu MF, Xing X, Lei S, et al. Electroacupuncture at Bilateral Zusanli Points (ST36) Protects Intestinal Mucosal Immune Barrier in Sepsis. Evid Based Complement Alternat Med. 2015;2015:639412.

14. Doig GS, Chevrou-Séverac H, Simpson F. Early enteral nutrition in critical illness: a full economic analysis using US costs. Clinicoecon Outcomes Res CEOR. 2013;5:429-436. 
15. Reintam Blaser A, Poeze M, Malbrain ML, et al. Gastrointestinal symptoms during the first week of intensive care are associated with poor outcome: a prospective multicentre study. Intensive Care Med. 2013;39:899-909.

16. Fang D. Experimental Study on the Effect of Rheum Officinale by Different Routes of Administration on Gastroin testinal Motility of Mice and Its Underlying Mechanism. Zhejiang Journal of Integrated Traditional Chinese \& Western Medicine. 2012.

17. Kim BJ, Kim H, Lee GS, et al. Effects of San-Huang-Xie-Xin-tang, a traditional Chinese prescription for clearing away heat and toxin, on the pacemaker activities of interstitial cells of Cajal from the murine small intestine. J Ethnopharmacol. 2014;155(1):744-752.

18. Yu ZX, Kuang Y, Tan YW, et al. [Case-control studies on debridement combined with external application of Mangxiao for the treatment of severe open injuries of limbs]. Zhongguo Gu Shang. 2009;22(3):196198.

19. Ma TT, Yu SY, Li Y, et al. Randomised clinical trial: an assessment of acupuncture on specific meridian or specific acupoint vs. sham acupuncture for treating functional dyspepsia. Aliment Pharmacol Ther. 2012;35(5):552-561.

20. Henry H, Marmy Conus N, Steenhout P, et al. Sensitive determination of D-lactic acid and L-lactic acid in urine by high-performance liquid chromatography-tandem mass spectrometry. Biomed Chromatogr. 2012;26(4):425-428.

21. Sanger GJ. Motilin receptor neuropharmacology: revised understanding. Curr Opin Pharmacol. 2012;12(6):641-646.

22. Takahashi T. Effect and mechanism of acupuncture on gastrointestinal diseases. Int Rev Neurobiol. 2013;111:273-294.

23. Fang. Therapeutical Effects and Mechanism of Rhubarb on Multiple Organ Dysfunction Ayndrome in Critical Illness. Chinese Journal of Modern Applied Pharmacy. 2012;29:953-956.
24. Zhang D, Li N, Dong L, et al. Evaluation of clinical application of ESICM acute gastrointestinal injury grading system: a single-center observational study. Chin Med J. 2014;127(10):1833-1836.

25. Levy MM, Fink MP, Marshall JC, et al. 2001 SCCM/ESICM/ACCP/ ATS/SIS International Sepsis Definitions Conference. Intensive Care Med. 2003;29(4):530-538.

26. de Haan JJ, Pastille E, Wirsdörfer F, et al. Lipid-rich enteral nutrition improves the defense against an opportunistic infection during polymicrobial sepsis. Shock. 2014;41(2):109-114.

27. Elke G, Kuhnt E, Ragaller M, et al. Enteral nutrition is associated with improved outcome in patients with severe sepsis. A secondary analysis of the VISEP trial. Med Klin Intensivmed Notfmed. 2013;108(3):223233

28. Zhu WF. Diagnostics of traditional Chinese medicine. China: Shanghai Science \& Technology Publishers; 1995.

29. Reintam Blaser A, Malbrain ML, Starkopf J, et al. Gastrointestinal function in intensive care patients: terminology, definitions and management. Recommendations of the ESICM Working Group on Abdominal Problems. Intensive Care Med. 2012;38(3):384-394.

30. Keller J. [Gastrointestinal dysfunction]. Deutsche medizinische Wochenschrift. 2012;137:2643-2656.

31. Reintam A, Parm P, Redlich U, et al. Gastrointestinal failure in intensive care: a retrospective clinical study in three different intensive care units in Germany and Estonia. BMC Gastroenterol. 2006;6:19.

32. Reintam A, Parm P, Kitus R, et al.Gastrointestinal failure score in critically ill patients: a prospective observational study. Crit Care. 2008;12(4):R90.

33. Sun JK, Li WQ, Ni HB, et al. Modified gastrointestinal failure score for patients with severe acute pancreatitis. Surg Today. 2013;43(5):506-513. 